\title{
Une maladie du génome mitochondrial : l'encéphalomyopathie familiale
}

Les protéines des mitochondries sont pour la plupart sous la dépendance de gènes du noyau. Toutefois la synthèse de certaines d'entre elles est dirigée par l'ADN propre des mitochondries ; cet «ADNmt » code chez l'homme pour 13 polypeptides, du système des phosphorylations oxydatives et pour des ADN ribosomiques et de transfert. Génétiquement, le caractère essentiel de l'ADNmt est d'être à transmission uniquement maternelle. Les myopathies mitochondriales, qu'on reconnaît de plus en plus fréquemment, ont pris une place importante en pathologie musculaire. Plus rares sont les cas où les symptômes neurologiques prédominent. L'épilepsie myoclonique associée au syndrome de ragged-red muscle fibers (fibres musculaires rouges en lambeaux), décrite en 1980 par Fukuhara et al. [1] atteint simultanément système nerveux central et muscle strié

Wallace et al. [2], aux USA, ont étudié en détail une famille dont la proposita présentait le tableau clinique complet, incluant, outre l'épilepsie myoclonique et une myopathie, surdité, ataxie et démence. En tout neuf sujets sur 12 avaient des symptômes anormaux de gravité variable. Cette famille montrait les caractéristiques qu'on peut attendre d'une anomalie de l'ADN mitochondrial. La transmission était uniquement maternelle: les neuf sujets atteints étaient la grand-mère, ses quatre enfants (trois filles et un garçon) et les quatre enfants de ses filles ; les trois sujets indemnes étaient les enfants du fils. Le deuxième caractère était l'existence de défauts des phosphorylations oxydatives, en conformité avec ce que fait prévoir une lésion de l'ADNmt. Le niveau du déficit dans le fonctionnement des complexes biochimiques de la phosphorylation oxydative varie entre les différents malades, parallèlement $\mathrm{m} / \mathrm{s} n^{\circ} 2$ vol. 5, féurier 89 aux différences de gravité clinique. L'interprétation de cette variabilité est qu'une mutation a frappé une partie seulement de l'ADNmt, ce que les auteurs appellent une mutation hétéroplastique. Cet $\mathrm{ADN}$ est présent sous forme de nombreuses copies dans chaque cellule, et la répartition de l'ADN normal et muté varie selon les individus. Enfin l'atteinte des différents organes se fait séquentiellement, en fonction du degré de dépendance de chaque tissu vis-à-vis de la production d'énergie par les mitochondries (effet de seuil). Le système nerveux central est frappé le premier, suivi des fibres musculaires de type I ou oxydatives puis du cœur, du rein, du foie

Le mécanisme moléculaire de la maladie n'a été que partiellement élucidé. Il diffère de celui d'observations de myopathies mitochondriales dans lesquelles on a trouvé une délétion, uniquement musculaire, de l'ADNmt (Holt et al., voir $\mathrm{m} / \mathrm{s} n^{\circ} 7$, vol. 4, p. 457) [3]. En effet Wallace et al. n'ont trouvé aucune délétion de l'ADNmt. De même, l'étude de la biosynthèse des protéines dans des lignées lymphoblastoïdes des malades n'a pas montré d'anomalies caractéristiques. Les auteurs concluent à la probabilité d'une mutation ponctuelle - soit d'une des sous-unités du complexe des phosphorylations oxydatives, soit d'un des ARN ribosomiques ou de transfert - qui rendrait inactives plusieurs sous-unités. Le type «hétéroplastique», créant deux populations d'ADNmt, l'une mutée et l'autre non, contraste avec les observations faites par les mêmes auteurs [4] dans

\footnotetext{
* V.A. McKusick a publié un catalogue des maladies à transmission mendélienne qui fait autorité: McKusick VA. Mendelian inheri. tance in man. The Johns Hopkins Univ. Press, 6th edition, 1983. Il est d'usage fréquent de donner ce numéro "McKusick" des maladies génétiques rares.
}

la neuropathie optique héréditaire de Leber (McKusick no $30890^{*}$ ) dans laquelle une atteinte sporadique de certains individus dans la lignée maternelle se traduit par une neuropathie optique tardive sans anomalies de l'oxydation dans le muscle. Ils ont trouvé dans cette maladie une mutation faux-sens homoplastique (affectant la totalité de l'ADNmt) dans un des gènes du complexe, celui de la sous-unité 4 de la NADH déshydrogénase (arg $\rightarrow$ his). Le diagnostic en est facile car un site de restriction de l'enzyme Sfa $\mathrm{Nl}$ est éliminé. Leur interprétation est que, dans la maladie de Leber, la variabilité clinique serait due à des facteurs supplémentaires, génétiques ou non, qui moduleraient les effets de la mutation.

En conclusion, les auteurs estiment que beaucoup de formes discrètes de maladies mitochondriales doivent encore passer inaperçues ; il importe de les reconnaître par l'examen approfondi de la lignée maternelle et l'emploi de critères biochimiques précis.

J.-C. D.

1. Fukuhara N, Tokiguchi S, Shirakawa K Tsubaki T. Myoclonus epilepsy associated with ragged-red fibers (mitochondrial abnormalities) : disease entity or syndrome? J Neurol Sci $1980 ; 47: 117-33$

2. Lestienne P, Ponsot G. Kearns-Sayre syndrome with muscle mitochondrial deletion. Lancet 1988 ; i : 885.

3. Wallace DC, Zheng X, Lott MT, et al Familial mitochondrial encephalomyopathy (MERRF) : genetic; pathophysiological, and biochemical characterization of a mitochon drial DNA disease. Cell 1988; 55 : 601-10.

4. Wallace DC, Singh G, Lott MT, et al. Mitochondrial DNA mutation associated with Leber's hereditary optic neuropathy Science 1988 ; 242 : 1427-30. 